\title{
Identification of PCAG1 as a novel prostate cancer-associated gene
}

\author{
YONGQING LAI ${ }^{1,2}$, ZUHU YU ${ }^{1,2}$, YADONG WANG ${ }^{1,2}$ and JIONGXIAN YE ${ }^{1,2}$ \\ ${ }^{1}$ Department of Urology, Peking University Shenzhen Hospital; ${ }^{2}$ Guangdong and Shenzhen Key Laboratory of \\ Male Reproductive Medicine and Genetics, Institute of Urology, Peking University Shenzhen Hospital, \\ Shenzhen PKU-HKUST Medical Center, Shenzhen, P.R. China
}

Received September 17, 2012; Accepted December 11, 2012

DOI: $10.3892 / \mathrm{mmr} .2012 .1249$

\begin{abstract}
The aim of the present study was to identify a new prostate cancer-associated gene and analyze its expression pattern. Comprehensive expression analysis of expressed sequence tags (ESTs) and microarray data and serial analysis of gene expression (SAGE) were conducted to screen in silico for candidate prostate cancer-associated genes. Reverse transcription (RT)-PCR was performed to validate prostate cancer specificity. Prostate cancer-associated gene 1 (PCAG1) was identified. The expression of PCAG1 mRNA and protein was evaluated in common human normal tissues, common malignant tumors, prostate adenocarcinoma and paired adjacent normal prostate tissues. An immunofluorescence assay was conducted to determine the subcellular location of PCAG1. PCAG1 mRNA was absent in the 15 pooled normal tissues (including normal prostate tissue) but registered at low levels in the spleen tissue (+). By contrast, PCAG1 mRNA was significantly higher than in the adjacent normal tissues in each of the 14 cases of prostate cancer, with 50\% scoring a high degree of expression (+++). Of the 32 types of normal tissues, 29 (including normal prostate tissue) demonstrated negative PCAG1 protein staining while the remaining tissues of the adrenal gland, parathyroid gland and liver expressed low levels. While 18/20 cases of prostate adenocarcinoma showed positive expression results, PCAG1 protein expression in the remaining types of cancer was scarce when present at all; only 41/380 other cancer cases demonstrated positive results at a low level. The most substantial PCAG1positive expression results were identified by cytoplasmic staining in $36 / 38$ prostate adenocarcinoma cases, with 10
\end{abstract}

Correspondence to: Professor Yongqing Lai, Department of Urology, Peking University Shenzhen Hospital, Lianhua Road 1120, Shenzhen, Guangdong 518036, P.R. China

E-mail: yqlord@163.com

Professor Jiongxian Ye, Hong Kong University Shenzhen Hospital, Baishi Road, Shenzhen 518053, P.R. China

E-mail: yjx66@126.com

Key words: prostate cancer, prostate cancer-associated gene 1, bioinformatics cases showing high expression levels, 20 showing medium levels and 6 showing low levels. In the paired adjacent normal prostate tissues, only $3 / 38$ cases showed low level positive staining, while $35 / 38$ cases were negative. Immunofluorescent staining of the human prostate cancer PC 3 cell line showed positive PCAG1 expression results in the mitochondria. The present study demonstrated that while PCAG1 mRNA was highly expressed in prostate cancer tissues, it was almost absent in all common normal tissues and paired adjacent normal prostate tissues. Furthermore, PCAG1 protein was also highly expressed in prostate cancer tissues, while few common normal tissues, other common malignant tumors and paired adjacent normal prostate tissues had even low levels of expression. Clarification of the function and transcriptional mechanism of PCAG1 may aid the elucidation of the mechanisms of carcinogenesis and progression of prostate cancer. The unique expression pattern of PCAG1 suggests its potential in certain clinical applications.

\section{Introduction}

The incidence of prostate cancer has increased over several decades such that it is now the most commonly diagnosed cancer among males in Europe and the USA $(1,2)$. The exact mechanisms for the carcinogenesis and progression of prostate cancer are not yet clear. Several prostate cancer-associated genes have been identified to have significant roles in these processes (3). The inadequacies of current diagnostic, prognostic, predictive and therapeutic markers have created a requirement for novel prostate cancer-associated genes $(4,5)$, therefore it is vital to search for these new genes.

In the present study, we performed comprehensive expression analysis of expressed sequence tags (ESTs), microarray data and serial analysis of gene expression (SAGE) to screen candidate prostate cancer-associated genes in silico. Prostate cancer-associated gene 1 (PCAG1) was identified. mRNA and protein expression levels were evaluated in human common normal tissues, common malignant tumors, prostate cancers and paired adjacent normal prostate tissues. PCAG1 mRNA was highly expressed in prostate cancer tissues, while few normal tissues showed a low level of expression. tissues with low level of expression. PCAG1 protein was highly expressed in prostate cancer tissues, while few common normal tissues, 
other common malignant tumors and paired adjacent normal prostate tissues demonstrated even lower levels of expression. Furthermore, PCAG1 protein was mainly shown to be localized within the cytoplasm and mitochondria using immunofluorescent staining.

\section{Materials and methods}

Tissue specimens. Paired prostate adenocarcinoma and adjacent normal tissues were collected for RT-PCR from 14 patients who underwent surgery between February 2009 and December 2010. The fresh tissues were immediately immersed in RNAlater solution (Qiagen, Hilden, Germany) following surgical resection, stored at $4^{\circ} \mathrm{C}$ overnight to allow thorough penetration of the tissue and then frozen at $-80^{\circ} \mathrm{C}$ until RNA extraction was performed. In addition, 38 paired paraffin-embedded samples of renal cell carcinoma and adjacent normal renal tissue that had been collected between 2008 and 2010 were stored for immunohistochemical assay. The patients had not received any therapy for prostate cancer prior to surgery. All samples were pathologically confirmed. Collection and use of patient samples were reviewed and approved by the Hospital Ethics Committees and informed consent was obtained from all patients.

Multiple organ normal tissue microarrays (catalog number, FDA999a) and high-density multiple organ tumor and normal tissue microarrays (catalog number, MC5003) were purchased from Biomax (Rockville, MD, USA). The FDA999a tissue microarray contained 3 cases of 32 common types of normal human organs, including tissue from the cerebrum, cerebellum, adrenal gland, ovary, pancreas, parathyroid gland, hypophysis, testis, thyroid gland, breast, spleen, tonsil, thymus, bone marrow, lung, cardiac muscle, esophagus, stomach, small intestine, colon, liver, salivary gland, kidney, prostate, endometrium, uterine cervix, skeletal muscle, skin, peripheral nerve, mesothelium, retina and larynx. Each sample type was obtained from 3 normal human individuals and had a single core per case. The MC5003 tissue microarray contained 20 common types of human cancer tissues and had a single core per case. These tissues were from cases of bladder urothelial carcinoma, brain astrocytoma/cerebrum glioblastoma, breast invasive ductal carcinoma, cervical squamous cell carcinoma, colon adenocarcinoma, esophageal carcinoma, head and neck carcinoma, kidney clear cell carcinoma, liver hepatocellular carcinoma, lung carcinoma, lymph node diffuse B cell lymphoma and Hodgkin disease, malignant melanoma, ovary carcinoma, pancreas adenocarcinoma, prostate adenocarcinoma, soft tissue fibrosarcoma, stomach adenocarcinoma, testicular cancer, thyroid papillary carcinoma and uterus endometrioid adenocarcinoma.

Perl programming to screen candidate genes in silico. To screen out new prostate cancer-associated genes in silico, the following steps were applied. Firstly, a secondary classification database for EST libraries was generated based on the Cancer Genome Anatomy Project (CGAP) information on EST libraries (6). The CGAP EST libraries were classified into two classes: libraries from non-fetal, non-germinal and non-placental normal tissues (NTs) and libraries from prostate cancer. Secondly, Unigene clusters with $<20$ ESTs from NT libraries and $>2$ ESTs from
TCC libraries were screened out. Thirdly, the frequency of the best SAGE tag in NT for each candidate gene was counted based on CGAP SAGE data and Unigene clusters, while $<20$ SAGE tags from NTs were retained for further analysis. Lastly, the candidate genes were analyzed manually with Affymetrix HG-U133A/B microarray data of normal tissues downloaded from the University of California at Los Angeles public core.

RT-PCR. Pooled cDNA from 16 normal tissues was obtained from Clontech (Palo Alto, CA, USA). Total RNA from cancerous tissues was extracted using the TRIzol solution (Invitrogen, Carlsbad, CA, USA) according to the manufacturer's instructions and with RNase-free DNase I used to remove the DNA contamination. Total RNA $(2 \mu \mathrm{g})$ was treated with M-MLV Reverse Transcriptase (Fermentas, Pittsburgh, PA, USA) to synthesize the first-strand cDNA according to the manufacturer's recommendations. The cDNA was then subjected to RT-PCR for evaluation of the relative mRNA levels of PCAG1 and GAPDH (as an internal control) with the corresponding primer pairs: PCAG1 sense, 5'-CCGCAGAAGAACAGAAGACC-3' and antisense, 5'-CAGAGAAACCATGGGCACTT-3'; GAPDH sense, 5'-CACCAGGGCTGCTTTTAACTC-3' and antisense, 5'-GAAGATGGTGATGGGATTTC-3'. The amplification conditions were $50^{\circ} \mathrm{C}(2 \mathrm{~min})$ and $95^{\circ}(2 \mathrm{~min})$ for 1 cycle, then $95^{\circ} \mathrm{C}(15 \mathrm{sec}), 55^{\circ} \mathrm{C}(30 \mathrm{sec})$ and $72^{\circ} \mathrm{C}(40 \mathrm{sec})$ for 35 cycles. Each PCR product was separated by $7 \%$ polycrylamide gel electrophoresis and visualized with ethidium bromide, excised from each gel and dissolved overnight using $0.5 \mathrm{~N}$ quaternary ammonium hydroxide in toluene (Soluenen 350; Packard Instruments Co., Meriden, CT, USA). After the addition of scintillation fluid, the samples were measured by scintillation spectroscopy. The mRNA results are expressed as a ratio of counts per min (cpm) of PCAG1 divided by GAPDH and were assigned to a semiquantitative score (+ to +++$)$.

Generation of anti-PCAGl antibody. Full-length cDNA of PCAG1 was inserted into pET23d (Novagen, Darmstadt, Germany) and expressed in Escherichia coli. The polypeptide was purified by applying it to a Ni-NTA resin (Qiagen) and used to immunize rabbits. Rabbit antiserum was then purified by applying it to the polypeptide column.

Immunohistochemistry assay. Immunohistochemistry procedures were performed with classical protocols. In brief, paraffin-embedded specimens were cut into 5- $\mu \mathrm{m}$ sections and baked at $65^{\circ} \mathrm{C}$ for $30 \mathrm{~min}$. The sections were deparaffinized with xylene and rehydrated. Sections were submerged in $0.01 \mathrm{~mol}$ citrate antigenic retrieval buffer $(\mathrm{pH} 6.0)$ and microwaved for antigenic retrieval. The sections were then treated with $3 \%$ hydrogen peroxide in methanol to quench the endogenous peroxidase activity, followed by incubation with $10 \%$ bovine serum albumin to block the non-specific binding. The PCAG1 protein was detected using a rabbit polyclonal antibody against PCAG1. The specimens were incubated with anti-PCAG1 antibody $(1: 100)$ overnight at $4^{\circ} \mathrm{C}$. The negative immunohistochemical control procedure included replacement of the primary antibodies by antibody diluent.

Subsequent to being washed in PBS, sections were treated with MaxVision HRP-Polymer anti-Rabbit IHC kit (Maixin Bio, Fujian, China) at $37^{\circ} \mathrm{C}$ for $15-20 \mathrm{~min}$. The tissue sections 
Table I. Expression of PCAG1 in various malignant tumor tissues evaluated with IHC.

\begin{tabular}{|c|c|c|c|c|c|c|}
\hline \multirow[b]{2}{*}{ Malignant tumors } & \multicolumn{6}{|c|}{ Expression } \\
\hline & Positive (rate, $\%$ ) & High & Medium & Low & Negative & Total \\
\hline Bladder urothelial carcinoma & $0 / 20(0)$ & 0 & 0 & 0 & 20 & 20 \\
\hline Brain astrocytoma/cerebrum glioblastoma & $6 / 20(30)$ & 0 & 0 & 6 & 14 & 20 \\
\hline Breast invasive ductal carcinoma & $4 / 20(20)$ & 0 & 0 & 4 & 16 & 20 \\
\hline Cervix squamous cell carcinoma & $0 / 20(0)$ & 0 & 0 & 0 & 20 & 20 \\
\hline Colon adenocarcinoma & $5 / 20(25)$ & 0 & 0 & 5 & 15 & 20 \\
\hline Esophageal carcinoma & $0 / 20(0)$ & 0 & 0 & 0 & 20 & 20 \\
\hline Head and neck carcinoma & $5 / 20(25)$ & 0 & 0 & 5 & 15 & 20 \\
\hline Kidney clear cell carcinoma & $1 / 20(5)$ & 0 & 0 & 1 & 19 & 20 \\
\hline Liver hepatocellular carcinoma & $8 / 20(40)$ & 0 & 0 & 8 & 12 & 20 \\
\hline Lung cancer & $3 / 20(15)$ & 0 & 0 & 3 & 17 & 20 \\
\hline B cell lymphoma and Hodgkin disease & $0 / 20(0)$ & 0 & 0 & 0 & 20 & 20 \\
\hline Malignant melanoma & $0 / 20(0)$ & 0 & 0 & 0 & 20 & 20 \\
\hline Ovary carcinoma & $5 / 20(25)$ & 0 & 0 & 5 & 15 & 20 \\
\hline Pancreas adenocarcinoma & $0 / 20(0)$ & 0 & 0 & 0 & 20 & 20 \\
\hline Prostate adenocarcinoma & $18 / 20(90)$ & 6 & 7 & 5 & 2 & 20 \\
\hline Soft tissue fibrosarcoma & $0 / 20(0)$ & 0 & 0 & 0 & 20 & 20 \\
\hline Stomach adenocarcinoma & $2 / 20(10)$ & 0 & 0 & 2 & 18 & 20 \\
\hline Testicular cancer & $2 / 20(10)$ & 0 & 0 & 2 & 18 & 20 \\
\hline Thyroid papillary carcinoma & $0 / 20(0)$ & 0 & 0 & 0 & 20 & 20 \\
\hline Uterus endometrioid adenocarcinoma & $0 / 20(0)$ & 0 & 0 & 0 & 20 & 20 \\
\hline
\end{tabular}

PCAG1, prostate cancer-associated gene 1; IHC, immunohistochemistry.

were immersed in 3-amino-9-ethylcarbazole, counterstained with Mayer's hematoxylin, dehydrated and mounted in a crystal mount.

The degree of immunostaining of formalin-fixed, paraffin-embedded sections was reviewed and scored by two independent observers. The intensity of the staining varied from weak to strong. The staining intensity was graded according to the mean optical density method (7-9): 0 , no staining; 1 , weak staining (light yellow); 2 , moderate staining (yellow brown); and 3, strong staining (brown).

Immunofluorescence assay. The PC3 cells were fixed in 4\% paraformaldehyde, permeabilized with $0.2 \%$ Triton X-100 and then incubated with anti-PCAG1 antibody at a concentration of $20 \mathrm{mg} / \mathrm{ml}$ for $1 \mathrm{~h}$ at room temperature, followed by an incubation with anti-rabbit IgG-FITC (Santa Cruz Biotechnology Inc., Santa Cruz, CA, USA). Intracellular localization of the PCAG1 protein was examined under fluorescent microscopy.

Statistical analysis. All quantitative data were analyzed using Student's t-test. Statistical analysis was performed using the SPSS 17.0 package and $\mathrm{P}<0.05$ was considered to indicate a statistically significant difference.

\section{Results}

Results of in silico screening and RT-PCR evaluation. A total of 45 candidate clusters were first screened out by perl programming based on EST data; after secondary analysis using SAGE and microarray data, the result was narrowed to twelve clusters with reconfirmed low expression in normal tissues. The twelve clusters were ranked according to the number of ESTs from prostate cancer and RT-PCR was performed to evaluate the prostate cancer specificity. In the first five genes evaluated, C12orf62 (chromosome 12 open reading frame 62, Gene ID: 84987) showed high specificity for prostate cancer and we temporarily named it prostate cancerassociated gene 1 (PCAG1).

PCAG1 mRNA expression in normal tissues and prostate cancer tissues analyzed by RT-PCR. The transcriptional level of PCAG1 was determined by RT-PCR assays in all 16 normal human tissues (Clontech). Our results showed that PCAG1 mRNA was absent in the 15 pooled normal tissues (including normal prostate tissue) but registered at a low level in spleen tissue (+; Fig. 1A). By contrast, expression of PCAG1 mRNA in each of the 14 cases of prostate cancer was significantly higher than in the paired adjacent normal prostate tissues with approximately half of the cases scoring a high expression level (+++; Fig. 1B).

Immunohistochemical observations of PCAG1 protein expression in 32 types of normal tissue and 20 types of human common malignant tumor. In order to determine the expression of the PCAG1 protein, rabbit anti-PCAG1 antibody was used as described in Materials and methods. The expression 
A

PCAG1 expression in clontech 16 normal tissues

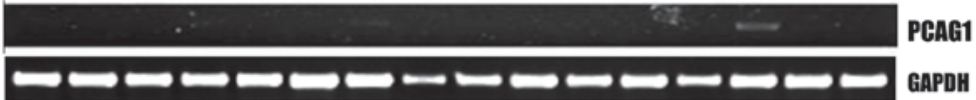

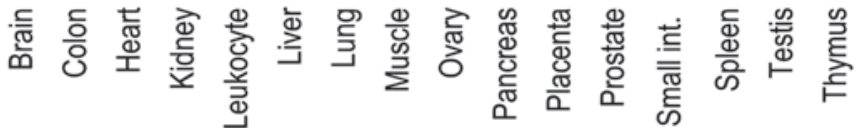

B

PCAG1 expression in prostate cancer tissues

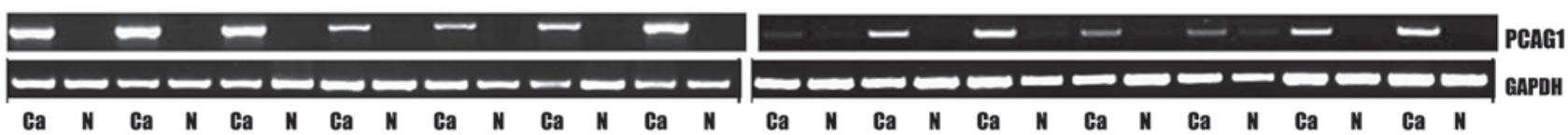

Figure 1. Expression of PCAG1 mRNA analyzed with RT-PCR. (A) Expression of PCAG1 mRNA in 16 normal tissues (Clontech). After 35 cycles of PCR, amplification was only observed in the spleen tissues (weak). (B) Expression of PCAG1 in prostate adenocarcinoma (Ca) and paired adjacent normal prostate tissues $(\mathrm{N})$. Expression of PCAG1 mRNA in all 14 cases of prostate cancers was significantly higher than in the paired adjacent normal prostate tissues. PCAG1, prostate cancer-associated gene 1; RT-PCR, reverse transcription PCR.

\section{PCAG1 expression in normal tissues}

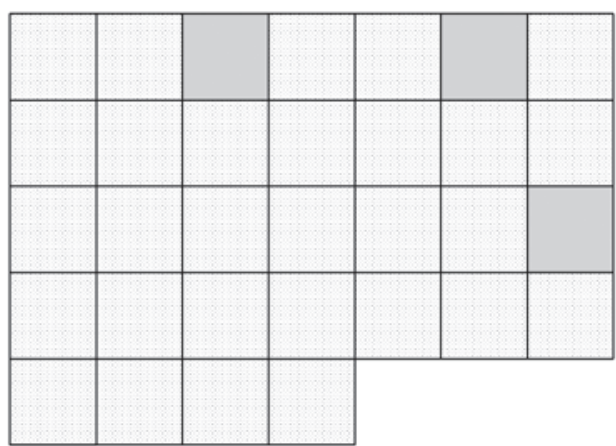

\section{C}

\section{PCAG1 in 38 cases of paied tissues}

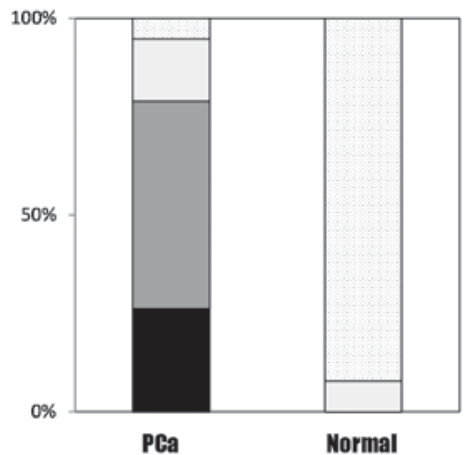

B

\section{PCAG1 expression in 20 types of common malignant tomors}
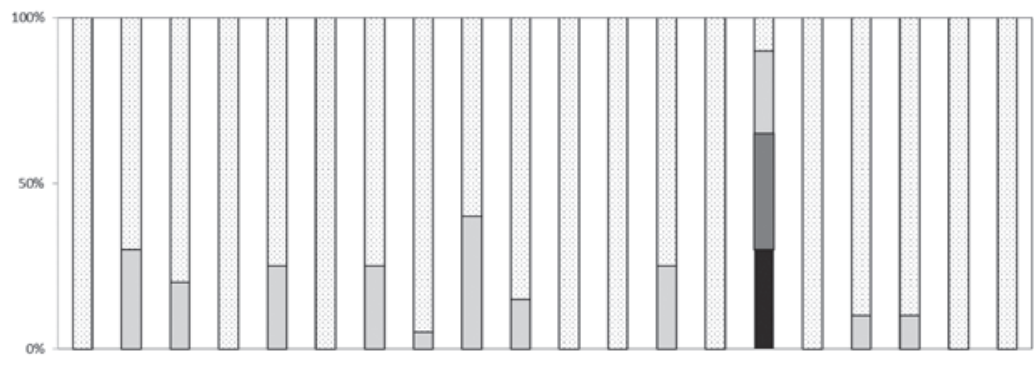

\section{Expression level}
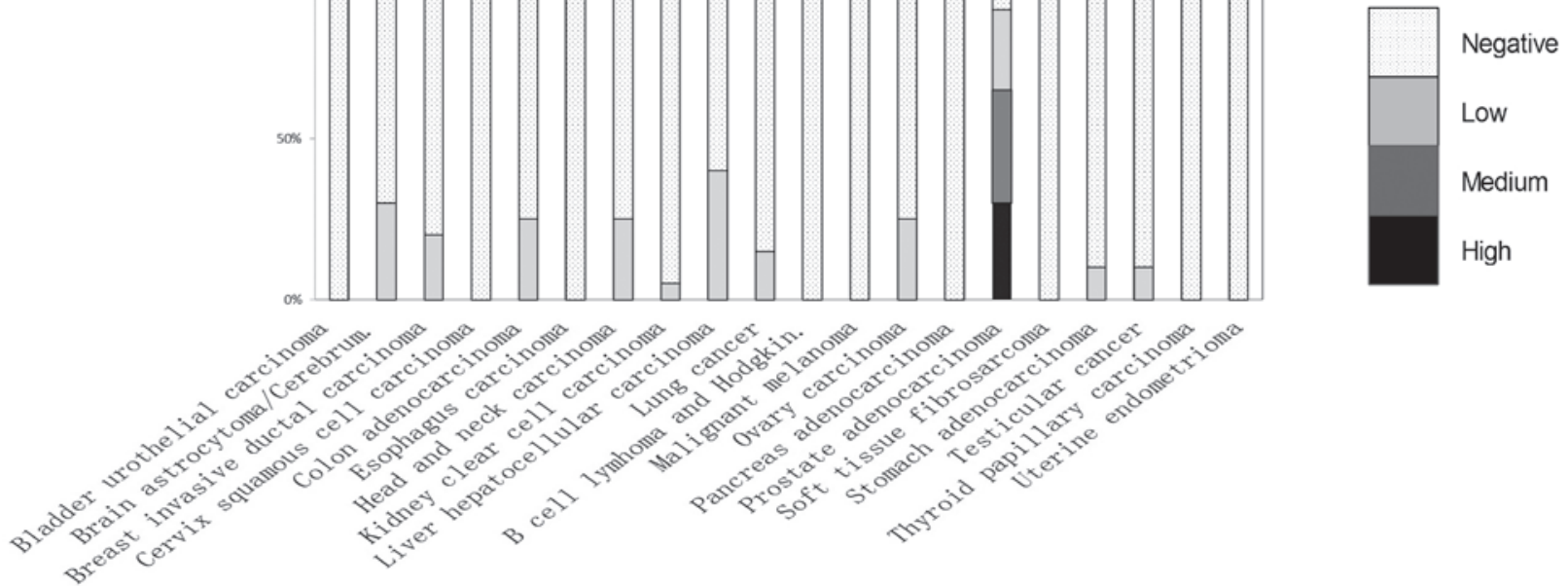

Figure 2. Expression of PCAG1 protein analyzed with immunohistochemistry (IHC). (A) Expression of PCAG1 protein in 32 types of normal human tissues. Each IHC dot (square) represents the expression level of PCAG1 in one tissue. From left to right and top to bottom, these dots represent cerebrum, cerebellum, adrenal gland, ovary, pancreas, parathyroid gland, hypophysis, testis, thyroid gland, breast, spleen, tonsil, thymus, bone marrow, lung, cardiac muscle, esophagus, stomach, small intestine, colon, liver, salivary gland, kidney, prostate, endometrium, uterine cervix, skeletal muscle, skin, peripheral nerve, mesothelium, retina and larynx. The expression levels of PCAG1 in the adrenal gland, parathyroid gland and liver were low while the other 29 tissues revealed negative results, including the normal prostate tissue. (B) Expression of PCAG1 protein in 20 types of common human malignant tumors. (C) Expression of PCAG1 protein in 38 cases of paired prostate adenocarcinoma (PCa) and paired adjacent normal prostate tissues. The expression in prostate adenocarcinoma revealed 10 cases showing high expression levels, 20 showing medium levels, 6 showing low levels and 2 with a negative result. Only 3/38 cases of paired adjacent normal prostate tissues showed positive (low) levels and 35/38 cases gave negative results. PCAG1, prostate cancer-associated gene 1. 
A

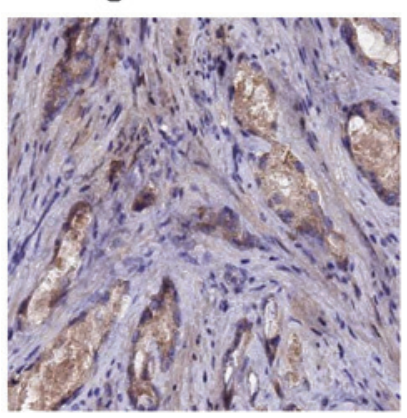

B Medium level in PCa

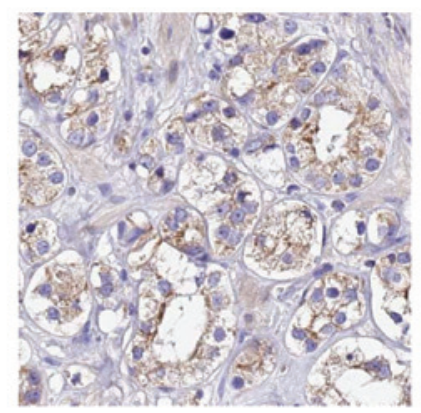

C

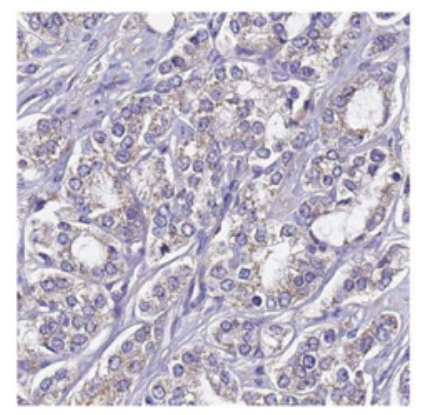

D Subcellular location with IF

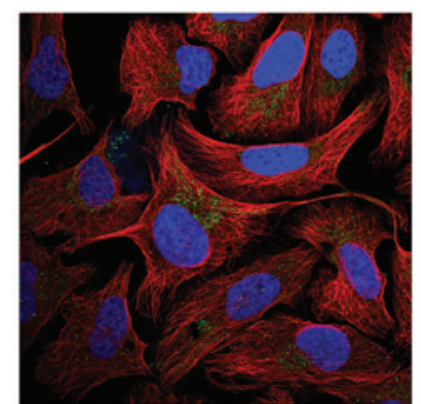

Figure 3. PCAG1 protein staining was mainly localized within the cytoplasm and mitochondria. (A) Strong PCAG1 staining in cancerous tissue (x400). (B) Moderate PCAG1 staining in cancerous tissue (x400). (C) Weak PCAG1 staining in cancerous tissue (x400). (D) Immunofluorescent staining for subcellular localization of PCAG1 protein in prostate cancer cell line PC3, showing positive PCAG1 staining in the mitochondria of PC3 cells. The cultures were fixed and stained for PCAG1 antibodies for cellular localization. The staining was restricted to the mitochondria and was in a characteristic pattern. The results are representative of three independent experiments. PCa, prostate adenocarcinoma; PCAG1, prostate cancer-associated gene 1; IF, immunofluorescent staining.

of PCAG1 protein was determined by immunohistochemistry in microarrays of 32 types of normal human tissues (Biomax; Fig. 2A). Of these tissues, 29/32 types (including normal prostate tissue) showed negative staining of PCAG1 while low levels were observed in the adrenal gland, parathyroid gland and liver tissues (Fig. 2A).

Expression of PCAG1 protein was also determined by immunohistochemistry in microarrays of 20 types of human common malignant tumors (Biomax; Fig. 2B and Table I). While $18 / 20$ cases of prostate adenocarcinoma showed positive results, PCAG1 protein expression in other types of cancer was scarce when present at all; only 41/380 other cancer cases demonstrated positive results at even a low level (Table I).

Immunohistochemical observations of PCAG1 protein expression in 38 cases of paired prostate adenocarcinoma and paired adjacent normal prostate tissues. In order to further determine the expression level of PCAG1 protein in prostate adenocarcinoma, immunohistochemistry assays were conducted in 38 cases of paired prostate adenocarcinoma and paired adjacent normal prostate tissues. As demonstrated in Fig. 2C, the most substantial PCAG1-positive expression results were identified by cytoplasmic staining in 36/38 prostate adenocarcinoma cases (Fig. 3A-C), with 10 cases showing high expression levels, 20 showing medium levels and 6 showing low levels. In paired adjacent normal prostate tissues, only $3 / 38$ cases demonstrated low level positive staining while $35 / 38$ cases were negative.

Immunofluorescent staining for subcellular localization of PCAG1 protein. To further determine the subcellular localization of the PCAG1 protein, we conducted an immunofluorescence assay in the human prostate cancer cell line PC3. As demonstrated in Fig. 3D, immunofluorescent staining of human prostate cancer PC3 cells for PCAG1 revealed a positive result in the mitochondria.

\section{Discussion}

In the present study, microarray data, ESTs and SAGE were used to increase the efficacy of expression analysis, as each technique had varying advantages and disadvantages. To screen out the prostate cancer-associated genes based on the databases of these three techniques, a specialized streamlined program was used prior to the expression patterns of the candidate genes being evaluated by immunohistochemistry and RT-PCR. Consequently, PCAG1 was identified. The results showed that screening in silico with this comprehensive strategy made the procedure to explore new prostate cancer-associated genes more efficient and accurate.

PCAG1 was temporarily named as PCAG1 in our screening procedure for prostate cancer-associated genes but is also designated as $\mathrm{C} 12$ orf62 (chromosome 12 open reading frame 62) $(10-12)$.

The full-length cDNA of PCAG1 was mapped to the 12q13.12 positive strand with two exons. Using Uniprot (13) and Nextprot (14), PCAG1 was predicted to be a transmembrane protein with a transmembrane domain of 22 amino acids. The present study showed that the PCAG1 protein was mainly localized within the cytoplasm and mitochondria.

The present study also demonstrated that PCAG1 mRNA and protein were expressed highly in prostate cancer but were almost absent in all normal tissues and other common malignant tumors. This indicates that PCAG1 has the features of prostate cancer-associated genes. Therefore, the functional significance of PCAG1 in prostate cancer deserves further investigation. The prostate cancer-specific expression of PCAG1 suggests a unique transcriptional regulation. Clarification of the function and transcriptional mechanism of PCAG1 may aid the elucidation of the mechanisms of carcinogenesis and progression of prostate cancer. The mechanisms behind prostate cancer carcinogenisis and progression may be resolved by a further elucidation of the functions and transcriptional mechanisms of PCAG1. Moreover, the unique expression pattern of PCAG1 suggests its potential as a marker of prostate cancer in early detection, forecasting disease severity, choosing treatments and monitoring responses to therapies.

In summary, the present study demonstrated that PCAG1 mRNA was highly expressed in prostate cancer tissues while being almost absent in all tested common normal tissues and paired adjacent normal prostate tissues. Furthermore, PCAG1 protein was also highly expressed in prostate cancer tissues while few common normal tissues, other common malignant 
tumors and paired adjacent normal prostate tissues showed even low levels of expression. Further analysis of the functions and transcriptional mechanisms of PCAG1 may aid in the understanding of the mechanisms for the carcinogenesis and progression of prostate cancer. The unique expression pattern of PCAG1 suggests its potential in certain clinical applications.

\section{Acknowledgements}

This study was supported by the National Natural Science Foundation of China (No. 81101922).

\section{References}

1. Bray F, Lortet-Tieulent J, Ferlay J, Forman D and Auvinen A Prostate cancer incidence and mortality trends in 37 European countries: an overview. Eur J Cancer 46: 3040-3052, 2010.

2. Siegel R, Ward E, Brawley O and Jemal A: Cancer statistics, 2011: the impact of eliminating socioeconomic and racial disparities on premature cancer deaths. CA Cancer J Clin 61: 212-236, 2011.

3. Shen MM and Abate-Shen C: Molecular genetics of prostate cancer: new prospects for old challenges. Genes Dev 24: 1967-2000, 2010.

4. Sardana G, Dowell B and Diamandis EP: Emerging biomarkers for the diagnosis and prognosis of prostate cancer. Clin Chem 54 1951-1960, 2008.

5. Shariat SF, Scherr DS, Gupta A, et al: Emerging biomarkers for prostate cancer diagnosis, staging, and prognosis. Arch Esp Urol 64: 681-694, 2011 (In English and Spanish).
6. Krizman DB, Wagner L, Lash A, Strausberg RL and Emmert-Buck MR: The Cancer Genome Anatomy Project: EST sequencing and the genetics of cancer progression. Neoplasia 1: 101-106, 1999.

7. Bao S, Ouyang G, Bai X, et al: Periostin potently promotes metastatic growth of colon cancer by augmenting cell survival via the Akt/PKB pathway. Cancer Cell 5: 329-339, 2004.

8. Chow WH, Dong LM and Devesa SS: Epidemiology and risk factors for kidney cancer. Nat Rev Urol 7: 245-257, 2010.

9. Tsuchiya A, Sakamoto M, Yasuda J, et al: Expression profiling in ovarian clear cell carcinoma: identification of hepatocyte nuclear factor-1 beta as a molecular marker and a possible molecular target for therapy of ovarian clear cell carcinoma. Am J Pathol 163: 2503-2512, 2003

10. Gerhard DS, Wagner L, Feingold EA, et al; MGC Project Team: The status, quality, and expansion of the NIH full-length cDNA project: the Mammalian Gene Collection (MGC). Genome Res 14: 2121-2127, 2004

11. Lamesch P, Li N, Milstein S, et al: hORFeome v3.1: a resource of human open reading frames representing over 10,000 human genes. Genomics 89: 307-315, 2007.

12. Strausberg RL, Feingold EA, Grouse LH, et al; Mammalian Gene Collection Program Team: Generation and initial analysis of more than 15,000 full-length human and mouse cDNA sequences. Proc Natl Acad Sci USA 99: 16899-16903, 2002.

13. UniProt Consortium: Reorganizing the protein space at the Universal Protein Resource (UniProt). Nucleic Acids Res 40: D71-D75, 2012.

14. Lane L, Argoud-Puy G, Britan A, et al: neXtProt: a knowledge platform for human proteins. Nucleic Acids Res 40: D76-D83, 2012. 